\title{
A Entrevista Motivacional em Adolescentes Usuários de Droga que Cometeram Ato Infracional
}

\author{
Motivational Interview with Adolescent Drug Users \\ who Have an Infringement
}

\author{
Ilana Andretta* \& Margareth da Silva Oliveira \\ Pontificia Universidade Católica do Rio Grande do Sul, Porto Alegre, Brasil
}

\begin{abstract}
Resumo
A drogadição na adolescência é um problema de saúde publica com alto custo para a sociedade, e há uma relação direta entre este e o cometimento de ato infracional. O objetivo deste estudo foi verificar a efetividade da Entrevista Motivacional (EM) em adolescentes que cometeram ato infracional, usuários de drogas. Utilizou-se a Entrevista Motivacional no grupo experimental e no grupo controle a Psicoeducação. Participaram do estudo 48 adolescentes: 27 no grupo da EM e 21 no grupo da Psicoeducação. O grupo da EM diminuiu consumo de maconha e tabaco e o grupo da Psicoeducação diminuiu o consumo de maconha e álcool. Com relação aos estágios motivacionais, independente do grupo, observou-se redução na média da pré-contemplação. As técnicas apresentaram resultados positivos em relação à diminuição do consumo de drogas e da média de pré-contemplação, entretanto, não houve diferença significativa entre as duas. Palavras-chave: Entrevista motivacional; Psicoeducação; Adolescente infrator; Drogas.
\end{abstract}

\begin{abstract}
Drug addiction in adolescence is a public health problem with high cost to the society, and there is a direct relationship between it and the commission of an infringement. The aim of this study was to evaluate the effectiveness of Motivational Interview (MS) in adolescent drug users who have committed an infringement. We used the Motivational Interview in the experimental group and the Psychoeducation in the control group. Forty eight adolescents participated of the study: 27 in the MS group and 21 in the group of Psychoeducation. The group of MS decreased consumption of marijuana and tobacco and the Psychoeducation group decreased the consumption of marijuana and alcohol. With respect to motivation, regardless of group settings, there was reduction in the average of pre-contemplation. The techniques presented positive results in terms of reducing drug use and the average of pre-contemplation. However, there was no significant difference between the two techniques.

Keywords: Motivational interview; Psychoeducation; Adolescent offenders; Drugs.
\end{abstract}

O uso ou abuso de drogas na adolescência é um problema de saúde pública, crônico, e recorrente, que requer atenção especial dos profissionais que trabalham na área da saúde. Há um crescimento nos índices de usuários de drogas nesta faixa etária, e a idade de experimentação está sendo cada vez mais precoce (Carlini, Galduroz, Notto, \& Napo, 2002). Os abusadores adolescentes experienciam uma variedade de problemas, incluindo rapidez na evolução do diagnóstico de abusadores para dependentes, e uma ocorrência maior de comorbidades relacionadas ao uso, bem como envolvimento com atos infracio-nais (Becker \& Curry, 2008; Martin \& Copeland, 2008), sendo esses os fatores que fazem com que os

*Endereço para correspondência: Pontifícia Universidade Católica do Rio Grande do Sul, Faculdade de Psicologia, Av. Ipiranga, 6681, prédio 11, Partenon, Porto Alegre, RS, Brasil, CEP 90619-900. Tel.: (51) 33203500. E-mails: ilana.andretta@pucrs.bre marga@pucrs.br profissionais da área da saúde, tentem buscar alternativas eficientes para intervenções que auxiliem na prevenção e resolução desta problemática (French et al., 2008).

Há relatos de pesquisas, com diversas técnicas que têm se mostrado eficazes para uso de drogas na adolescência: terapia familiar de modelo cognitivo, ecológico e funcional; intervenções breves, como comportamentais, cognitivo-comportamental e grupoterapia; treinamento de pais e alguns modelos integrados que combinam técnicas de mais de um modelo teórico, como a Entrevista Motivacional e técnicas cognitivo-comportamentais (Battjes et al., 2004; Becker \& Curry, 2008; Muck et al., 2001; Tait \& Hulse, 2003).

A escolha do tipo de tratamento está diretamente ligada ao perfil do usuário, a gravidade da dependência e ao tipo de estrutura social a que o mesmo está inserido (Dennis et al., 2002; Muck et al., 2001). Entretanto, alguns autores são unânimes ao afirmar que, quando se fala em adolescentes, usuários de drogas, que vêm encami- 
nhados para tratamento não por vontade própria, a chave para o sucesso do mesmo é a motivação (Tevyaw \& Monti, 2004); e há poucos estudos que avaliam a efetividade de intervenções nesta população (Andretta \& Oliveira, 2008; Diamond et al., 2002; Lincourt, Kuettel, \& Bombardier, 2002; Stein et al., 2006).

A Entrevista Motivacional (EM) é uma intervenção breve que tem se mostrado eficaz para aumentar a motivação para a mudança em diversos comportamentos e em diversos contextos, inclusive na adolescência (Andretta \& Oliveira, 2005; Brown et al., 2003; Colby et al., 2005; D’Amico, Miles, Stern, \& Meredith, 2008; Lawendowski, 1998; McCambridge \& Strang, 2004; Monti et al., 1999). É um modelo de intervenção baseado nos conceitos de ambivalência e prontidão para mudança. Ambivalência é entendida como o conflito existente entre o mudar e o permanecer no comportamento atual; e a prontidão para mudança é descrita em estágios que oscilam entre não ter consciência do problema (pré-contemplação), admitir que há um problema (contemplação), fazer algo para mudar (ação) e manter as mudanças alcançadas (manutenção) (McCambridge \& Strang, 2004).

O estilo do terapeuta motivacional é não confrontativo e não diretivo, embasado nas premissas de fornecer informações claras e precisas, dar responsabilidade ao cliente, proporcionar alternativas de mudança, expressar empatia e aumentar a auto-eficácia. Esses princípios da técnica se mostram adequados para trabalhar com adolescentes, inclusive os que vêm encaminhados para tratamento por terem cometido ato infracional, pois tendem a evitar a resistência comum ao processo de mudança (Andretta \& Oliveira, 2008; Lincourt et al., 2002; McCambridge \& Strang, 2004; Tevyaw \& Monti, 2004).

Esta técnica, também, tem se mostrado uma técnica adequada para predição ou para a facilitação de mudança, na medida em que pode ser combinada com outras técnicas, tendo como objetivo principal a conscientização dos clientes para os seus problemas, para depois buscar outras alternativas mais específicas, como resolução de problemas, treinamento de habilidades e modificação de pensamentos disfuncionais (Dennis et al., 2002; Dunn, Droesch, Johnston, \& Rivara, 2004; McCambridge \& Strang, 2004).

O objetivo principal deste estudo foi avaliar a efetividade da Entrevista Motivacional aplicada em adolescentes que cometeram ato infracional, usuários de drogas encaminhados para tratamento; além disso, identificar o padrão de consumo de drogas destes sujeitos, avaliar se houve diminuição de consumo de substâncias psicoativas e verificar se houve mudança no estágio motivacional após as intervenções.

\section{Método}

Este estudo é um estudo clínico, com avaliação e reavaliação antes e depois da intervenção em grupo controle e experimental. $\mathrm{O}$ fator em estudo foi a Entrevista
Motivacional, e os desfechos para avaliar a efetividade do estudo foram: (a) Diminuição do consumo de drogas: a freqüência e a quantidade registradas pelo protocolo da avaliação e reavaliação. (b) Motivação para mudança: o estágio motivacional e a prontidão para mudança foram verificados através da escala University Rhode Island Change Assessment (URICA).

\section{Amostra}

Os participantes desta pesquisa foram todos os sujeitos que foram atendidos no período de abril de 2004 a novembro de 2008, em uma clínica escola de uma faculdade de psicologia de uma universidade privada de Porto Alegre, encaminhados para tratamento para dependência química, por instituições que executam medidas sócioeducativas, que finalizaram o programa de atendimento.

A amostra foi escolhida por conveniência, com participantes do sexo feminino e masculino, com idades entre 12 e 19. Foram excluídos da amostra, participantes com síndrome de abstinência grave, com dificuldade cognitiva para entendimento dos instrumentos, e sujeitos que estavam fazendo outro tratamento psicoterapêutico.

O programa de atendimento foi iniciado por 135 adolescentes no período do estudo, sendo que: iniciaram algum tipo de intervenção 70 (51,9\%) adolescentes, sendo alocados $36(26,7 \%)$ no grupo da Entrevista Motivacional e $34(25,2 \%)$ no grupo da Psicoeducação. Dos adolescentes que foram alocados no grupo da Entrevista Motivacional, 4 (11,1\% do grupo) abandonaram o processo antes de concluir a intervenção, e outros 5 (13,9\% do grupo) antes da conclusão da reavaliação. Já no grupo da Psicoeducação, 11 (32,3\% do grupo) abandonaram o processo antes de concluir a intervenção, e outros 2 (5,9\% do grupo), antes de concluir a reavaliação. Desta forma, a amostra total deste estudo ficou resumida a 48 sujeitos, sendo que 27 deles do grupo da Entrevista Motivacional e 21 do grupo da Psicoeducação.

Os participantes foram alocados, para a formação dos grupos controle e de intervenção, por ordem de tempo, sendo que, de abril de 2004 a julho de 2006, foram alocados no grupo da EM e, de agosto de 2006 a novembro de 2008, foram alocados no grupo da Psicoeducação.

\section{Instrumentos}

Para a avaliação dos adolescentes, foi utilizado um protocolo para levantamento de dados sócio-demográficos da amostra, como sexo, idade, ocupação, escolaridade, familiar com uso de drogas e padrão do consumo de drogas como droga de eleição, freqüência, forma e tempo de uso; uma entrevista semi-estruturada contendo perguntas sobre todos os critérios diagnósticos do DSM-IV-TR (American Psychiatric Association [APA], 2002) para identificar dependência ou abuso de substâncias bem como diagnóstico de transtornos disrruptivos.

Foi utilizada a URICA, que se trata de uma escala que verifica os estágios motivacionais para mudança de comportamento; que foi validada para usuários de 
drogas ilícitas e mostrou bons resultados de confiabilidade, apresentando um coeficiente de Cronbach de 0,657 (Szupszynski \& Oliveira, 2008). Estes estágios são descritos em pré-contemplação, contemplação, ação e manutenção.

O Inventário de Depressão de Beck (BDI) e o Inventário de Ansiedade de Beck (BAI), têm por objetivo avaliar a intensidade dos sintomas de depressão e ansiedade, tanto em pacientes psiquiátricos como na população em geral.

O BDI trata-se de um instrumento composto por 21 categorias de sintomas considerados manifestações comportamentais ou cognitivas de depressão. As categorias são: pessimismo, sentimentos de fracasso, humor, insatisfação, sentimentos de culpa, sentimentos de punição, desejo de autopunição, autodepreciação, autoacusação, irritabilidade, isolamento social, crises de choro, distúrbio do sono, fatigabilidade, indecisão, inibição no trabalho, perda de apetite, perda de peso, peda da libido e preocupações somáticas.

Para cada categoria, é possível pontuar de 0 a 3 , sendo 0 a ausência total de sintomas e 3 presença dos sintomas de forma intensa. A soma da pontuação de todas as categoria é a pontuação total do instrumento. Pontuações totais até 9 são consideradas ausência de sintomas depressivos ou sintomas mínimos; de 10 a 18, sintomas leves a moderados; de 19 a 29, sintomas moderados a graves; de 30 a 63 sintomas graves. A versão do instrumento traduzida para o português foi validada por Cunha (2001) sendo o coeficiente de fidedignidade deste igual a 0,89 .

O BAI é um instrumento desenvolvido para avaliar intensidade de sintomas de ansiedade, dividida em 21 itens que avaliam os sintomas ansiosos evitando-se confusões com sintomas depressivos. Em cada item, o sujeito é questionado sobre quanto os sintomas ansiosos relatados o incomodaram na última semana. Para responder, o indivíduo escolhe, em uma escala de 0 (não a todas) a 3 (severamente) a alternativa que melhor explique a intensidade de seus sintomas. A versão em português deste instrumento foi validada por Cunha (2001), sendo o coeficiente de fidedignidade do mesmo igual a 0,92 .

\section{Procedimentos}

A equipe participante do projeto recebeu um treinamento padrão sobre todos os instrumentos de avaliação e com um profissional sênior na área sobre a Entrevista Motivacional. Durante toda a pesquisa, houve supervisão da equipe por profissionais especializados na avaliação e na técnica.

Os procedimentos para a coleta de dados deste estudo foram a aprovação pelo Comitê de Ética da Universidade, pelo Programa de Pós-Graduação da Faculdade e da Comissão Científica da faculdade em que ocorreu este estudo. Também, foi realizado um estudo piloto para verificação da viabilidade da execução deste projeto.

Para o início da coleta de dados, o adolescente deveria ser encaminhado pelas instituições especializadas a clínica escola, e, os pais ou responsáveis pelo adolescente, recebiam o Termo de Consentimento Livre e Esclarecido do projeto, optando por participar ou não da pesquisa. Após a assinatura do mesmo, o adolescente era avaliado através do protocolo padrão do serviço, com os instrumentos listados acima e por uma entrevista de anamnese com um dos pais ou informantes do sujeito, para verificação de dados de desenvolvimento e confirmação de informações. Posteriormente a avaliação, os participantes foram alocados, por ordem de tempo, nos grupos da EM e no grupo da Psicoeducação.

A Entrevista Motivacional (Miller \& Rollnick, 2001; Sampl \& Kadden, 2001), trata-se de um modelo de intervenção breve e focal, com o objetivo de aumentar a motivação para modificação de comportamentos. Neste estudo a intervenção com EM consistiu em cinco sessões estruturadas, baseadas no Cannabis Youth Treatment (Diamond et al., 2002), um programa de tratamento realizado nos Estados Unidos para adolescentes infratores. $\mathrm{Na}$ primeira sessão desta etapa, a finalidade era dar a devolução da avaliação para que o sujeito obtivesse informações sobre os prejuízos causados pelas drogas. A devolução é baseada no princípio do feedback, e tem por objetivo fornecer informações claras e desprovidas de julgamentos morais sobre a avaliação realizada anteriormente.

Na sessão dois, trabalhava-se com a técnica da Balança Decisacional (Knapp, Luz, \& Baldisserotto, 2001). Primeiramente, $\mathrm{o}$ adolescente apontava as vantagens que ele percebia, em sua vida cotidiana, em continuar usando drogas; e, em seguida, as desvantagens em continuar usando. O objetivo do terapeuta, neste momento, era assinalar para o adolescente que geralmente há mais desvantagens em continuar usando, que em parar de usar.

Na sessão seguinte, o tema era o Plano de Metas (Miller \& Rollnick 2001) para que o terapeuta ajudasse o adolescente a identificar quais seriam os possíveis obstáculos para alcançar seus objetivos e como ele poderia fazer para obter êxito. Na mesma sessão, era realizado o Treinamento de Situações de Risco (Diamond et al., 2002) com objetivo de desenvolver habilidades para situações inesperadas que pudessem ocorrer quando cessado o tratamento, aumentando o repertório de comportamentos adaptativos em situação de possível recaída.

Posteriormente, o objetivo foi aumentar atividades prazerosas e o suporte social. Nesta sessão o adolescente referia quais atividades gostaria de realizar, tais como esporte, como uma nova ocupação e como poderia tornálas uma prática constante em sua vida. Em relação ao suporte social, era referido quem seriam as pessoas com que ele poderia contar em situações de emergência, e em como fazer para buscar ajuda. Na última sessão desta etapa, estava previsto o fechamento do processo e o planejamento de situações de emergências que pudessem ocorrer. A avaliação do processo era feita pelos dois, apontando os pontos fortes e fracos do mesmo. Por último, era feito o encaminhamento terapêutico, caso fosse necessário, a outras instituições. 
O grupo da Psicoeducação recebeu duas sessões de informações acerca dos efeitos de drogas (Secretaria Nacional Antidrogas [SENAD], 2004), sem a devolução da avaliação, e três sessões de atendimento individual com técnicas cognitivo-comportamentais. A Psicoeducação, como tratamento para dependência química, é escolhida como um tratamento controle ético e apropriado metodologicamente, além de ser um tipo de intervenção padrão encontrado em diversos estudos com adolescentes (Smith, Sells, Rodman, \& Reynolds, 2006). Trata-se de uma intervenção que visa fornecer informações sobre drogas e sobre os problemas das mesmas (Kaminer, Burleson, \& Goldberger, 2002). Cabe salientar que a terceira, quarta e quinta sessões, eram iguais as do grupo da EM; entretanto, os modelos terapêuticos diferiram principalmente em relação à postura adotada pelo terapeuta.

Após 15 dias, ao término do tratamento, o adolescente era encaminhado novamente ao serviço para uma reavaliação, com objetivo de verificar se houve modificações no padrão de consumo e no estágio motivacional, após o término do processo. Caso necessário, isto é, se houvesse indicação, permaneciam em atendimento psicológico no mesmo serviço ou eram encaminhados para outro.

\section{Análise dos Dados}

Dados quantitativos serão descritos por média, desvio padrão ou mediana e intervalo interquartil, dependendo de sua distribuição; e dados qualitativos, por freqüência absoluta e relativa. Para verificar a homogeneidade e relação entre grupos, foi utilizado o teste Qui-quadrado para variáveis qualitativas e teste de comparação de médias $t$ de Student.

Para avaliar a interação entre tempo e grupos, em relação a cada estágio motivacional, utilizou-se a análise de variância, utilizando delineamento em medidas repetidas. Para avaliar a freqüência semanal de consumo de álcool, tabaco e maconha, entre a avaliação e a reavaliação, foi utilizado o Teste Não-Paramétrico Wilcoxon; e para comparar a variação da freqüência semanal de consumo de álcool, tabaco e maconha em relação aos grupos, foi utilizado o Teste Não-Paramétrico Mann-Whitney. Os dados foram compilados no software SPSS versão 11.0, e o nível de significância foi de $5 \%$.

\section{Resultados}

No período entre abril de 2004 e novembro de 2008, foram encaminhados para atendimento 135 . Destes, 86 (63,7\% da amostra total) adolescentes completaram o processo de avaliação, 55 (40,74\% da amostra total) adolescentes concluíram o processo de intervenção e 48 (35,55\% da amostra total) adolescentes finalizaram o processo de reavaliação, sendo 27 sujeitos no grupo da EM (56,3\% da amostra que finalizou a reavaliação) e 21 sujeitos no grupo da Psicoeducação (43,8\% da amostra que finalizou a reavaliação).
Para a avaliação da homogeneidade entre os grupos, verificou-se a distribuição das variáveis: $\operatorname{sexo}(p=0,574)$, estudar atualmente $(p=0,641)$, escolaridade $(p=0,729)$, idade $(p=0,343)$ e anos de estudo $(p=0,719)$, confirmando a semelhança da amostra.

No grupo da EM, a média de idade foi $16,37(D P=1,39)$, e a maioria dos sujeitos era do sexo masculino $(n=26$, $96,3 \%)$, que estudava atualmente $(n=25,92,6 \%)$, apresentando uma média de 7,56 $(D P=1,78)$ anos de estudo; apenas $6(22,2 \%)$ sujeitos trabalhavam no momento da avaliação, $11(40,7 \%)$ sujeitos relataram ser de classe baixa, $18(66 \%)$ cometeram o ato infracional de porte de drogas, $15(55,6 \%)$ sujeitos moravam com os pais e apenas $6(22,2 \%)$ tinham parceiro fixo.

A média de idade em anos, do primeiro uso de álcool foi 13,33 $(D P=1,44)$, de tabaco foi de 13,16 $(D P=1,77)$, e de maconha 13,48 $(D P=1,46)$. Com relação ao auto-relato sobre familiares com uso de drogas, $15(56,6 \%)$ sujeitos da amostra referiram ter algum familiar com uso de alguma droga ilícita, e $16(59,3 \%)$ sujeitos referiram algum familiar próximo com uso de tabaco e álcool.

No grupo da Psicoeducação, a média de idade foi de $17,24(D P=4,45)$, a maioria dos sujeitos era do sexo masculino $(n=19,90,5 \%)$, que estudava atualmente $(n=18,85,7 \%)$, com a média de anos de estudo de 7,76 $(D P=1,76)$, apenas $3(14,3 \%)$, sujeitos trabalhavam no momento da avaliação, $10(47,6 \%)$ sujeitos relataram ser de classe baixa, $5(23,7 \%)$ sujeitos cometeram o ato infracional de porte de drogas, $18(85,7 \%)$ sujeitos moravam com os pais e apenas $3(14,3 \%)$ sujeitos tinham parceiro fixo. A média de idade, em anos, do primeiro uso de álcool, foi de $13,89(D P=1,49)$, de tabaco foi de $13,00(D P=2,83)$ e de maconha foi de 14,05 $(D P=2,21)$. Com relação ao auto-relato sobre familiares com uso de drogas, 16 (59,3\%) sujeitos da amostra referiram ter algum familiar com uso de alguma droga ilícita(s), 21 (71,4\%) sujeitos, algum familiar com uso de álcool, e $19(62,2 \%)$ sujeitos referiram algum familiar próximo com uso de tabaco. Dados sobre freqüência de diagnósticos de dependência e transtornos disruptivos serão apresentados na Tabela 1.

Conforme os resultados obtidos na Tabela 1, com relação aos diagnósticos para o uso de substâncias, percebese uma homogeneidade dos grupos: nenhuma dependência de álcool, uma porcentagem um pouco maior para dependentes de tabaco no grupo da EM, porcentagens semelhantes em dependentes de maconha, crack e solventes; e, no grupo da Psicoeducação, um dependente de solvente.

No grupo da EM, há uma freqüência maior de comorbidades que no grupo da Psicoeducação para Transtorno de Déficit de Atenção e Hiperatividade (TDAH) e Transtorno Opositivo Desafiador (TOD); entretanto, as freqüências de Transtorno de Conduta (TC) foram semelhantes nos dois grupos.

A Tabela 2, descrita abaixo apresenta a mediana da freqüência semanal de álcool, tabaco e maconha, em cada um dos grupos. 
Tabela 1

Descrição da Freqüencia e Porcentagem de Resultados dos Participantes com Diagnóstico de Dependência de Substâncias e Transtornos Disruptivos

\begin{tabular}{|c|c|c|c|}
\hline & $\begin{array}{c}\text { Grupo } \operatorname{EM}(N=27) \\
(N=27)\end{array}$ & $\begin{array}{c}\text { Grupo Psicoeducação } \\
(N=21)\end{array}$ & $p$ \\
\hline Dependência de álcool & 0 & 0 & ** \\
\hline Abuso de álcool & $21(77,8 \%)$ & $11(52,4 \%)$ & 0,122 \\
\hline Dependência de tabaco & $21(77,8 \%)$ & $11(52,4 \%)$ & 0,122 \\
\hline Abuso de tabaco & 0 & $4(19 \%)$ & 0,031 \\
\hline Dependência de maconha & $18(66,7 \%)$ & $11(52,4 \%)$ & 0,380 \\
\hline Abuso de maconha & $9(33,3 \%)$ & $6(28,6 \%)$ & 0,764 \\
\hline Dependência de cocaína & 0 & $1(4,8 \%)$ & 0,426 \\
\hline Abuso de cocaína & $4(14,8 \%)$ & $1(4,8 \%)$ & 0,377 \\
\hline Dependência de crack & $2(7,4 \%)$ & $1(4,8 \%)$ & 1,00 \\
\hline Abuso de crack & $6(22,2 \%)$ & $1(4,8 \%)$ & 0,112 \\
\hline Dependência de solvente & $1(3,7 \%)$ & $1(4,8 \%)$ & 1,00 \\
\hline Abuso de Solvente & $2(7,4 \%)$ & $2(9,5 \%)$ & 1,00 \\
\hline Transtorno de Déficit de Atenção e Hiperatividade & $11(40,7 \%)$ & $2(9,5 \%)$ & 0,022 \\
\hline Transtorno Opositivo Desafiador & $7(25,9 \%)$ & $1(4,8 \%)$ & 0,115 \\
\hline Transtorno de Conduta & $10(37 \%)$ & $6(22,2 \%)$ & 0,761 \\
\hline Inventário de Ansiedade de Beck (Categoria Leve) & $13(48,1 \%)$ & $13(61,9 \%)$ & 0,548 \\
\hline Inventário de Depressão de Beck(Categoria Leve) & $13(48,1 \%)$ & $13(61,9 \%)$ & 0,639 \\
\hline
\end{tabular}

Nota. *Variáveis relacionadas através do teste Qui-quadrado. **Valor inexistente pela ausência de sujeitos dependentes de álcool em ambos os grupos.

Tabela 2

Consumo de Drogas em Medianas de Unidades para Cada Droga

\begin{tabular}{lcccccc}
\hline \multirow{2}{*}{ Consumo Semanal } & \multicolumn{2}{c}{ Grupo EM $(\mathrm{N}=27)$} & \multicolumn{3}{c}{ Grupo Psicoeducação $(\mathrm{N}=21)$} \\
\cline { 2 - 6 } & Antes & Depois & $\mathrm{p}$ & Antes & Depois & $\mathrm{p}$ \\
\hline $\begin{array}{l}\text { Álcool }(\mathrm{ml}) \\
\begin{array}{l}\text { Tabaco } \\
\text { (número de cigarros) }\end{array}\end{array}$ & $1500(200 ; 1500)$ & $350(0,83 ; 1500)$ & 0,173 & $11,55(0 ; 1500)$ & $11,78(0 ; 937,5)$ & 0,035 \\
$\begin{array}{l}\text { Maconha } \\
\text { (número de cigarros) }\end{array}$ & $3(0 ; 9)$ & $0(0 ; 1,5)$ & 0,013 & $6(1 ; 15)$ & $0,5(0 ; 1,75)$ & 0,020 \\
\hline
\end{tabular}

Nota. *Variáveis relacionadas através do teste Não-Paramétrico Wilcoxon.

Conforme os dados descritos da Tabela 2, no grupo da EM houve diminuição no consumo de tabaco $(p=0,001)$ e maconha $(p=0,013)$ depois da intervenção; e no grupo da Psicoeducação, houve redução do consumo de álcool $(p=0,035)$ e de maconha $(p=0,020)$ após a intervenção. É importante atentar que no resultado referente ao consumo de álcool do Grupo Psicoeducação, houve aumento da mediana, mas o intervalo interquartil diminuiu, caracterizando a diminuição do consumo. Entretanto, não houve diferença na variação do consumo de álcool $(p=0,190)$, de tabaco $(p=0,422)$ e de maconha $(p=0,732)$, comparando as duas técnicas. Resultados referentes ao estágio motivacional da Pré-contemplação serão apresentados na Tabela 3 .

Tabela 3

Média da Pontuação do Estágio Pré-contemplação com Análise de Variância em Medidas Repetidas

\begin{tabular}{lcccccc}
\hline \multirow{2}{*}{ Grupo } & \multicolumn{3}{c}{ Tempo } & & \multicolumn{2}{c}{ Total } \\
\cline { 2 - 5 } & \multicolumn{2}{c}{ Inicial } & \multicolumn{2}{c}{ Final } & & \\
& Média & Desvio-padrão & Média & Desvio-padrão & Média & Desvio-padrão \\
\hline Entrevista Motivacional & 13,78 & 4,49 & 11,52 & 3,81 & $12,65^{\mathrm{B}}$ & 4,28 \\
Psicoeducação & 17,19 & 4,65 & 16,10 & 5,50 & $16,64^{\mathrm{A}}$ & 5,06 \\
Total & $15,27^{\mathrm{a}}$ & 4,83 & $13,52^{\mathrm{b}}$ & 5,12 & 14,40 & 5,03 \\
\hline
\end{tabular}

Nota. Médias seguidas de letras maiúsculas distintas e médias seguidas de letras minúsculas distintas diferem significativamente através da Análise de Variância, utilizando delineamento em medidas repetidas, ao nível de significância de 5\%. 
Os dados descritos na Tabela 3 mostram que a média de valor da pontuação do estágio Pré-contemplação, independente de grupo, foi significativamente maior no tempo inicial que no tempo final $(F=4,99, p=0,030)$. Em relação aos grupos, independente do tempo, a média da pontuação do grupo Psicoeducação foi significativamente maior que do grupo da $\operatorname{EM}(F=13,06, p=0,001)$. Não houve interação significativa entre os fatores tempo e grupo $(F=0,60, p=0,442)$. Os Resultados referentes ao estágio motivacional da Contemplação serão apresentados na Tabela 4.

Tabela 4

Média da Pontuação do Estágio Contemplação com Análise de Variância em Medidas Repetidas Complementada pelo Teste de Comparações Múltipla de Tukey

\begin{tabular}{lcccccc}
\hline \multirow{2}{*}{ Grupo } & \multicolumn{2}{c}{ Inicial } & \multicolumn{2}{c}{ Tempo } & \multicolumn{2}{c}{ Total } \\
\cline { 2 - 7 } & Média & Desvio-padrão & Média & Desvio-padrão & Média & Desvio-padrão \\
\hline Entrevista Motivacional & $25,44^{\mathrm{Aa}}$ & 3,00 & $23,52^{\mathrm{Aa}}$ & 4,70 & 24,48 & 4,03 \\
Psicoeducação & $21,14^{\mathrm{Ba}}$ & 4,82 & $21,67^{\mathrm{Aa}}$ & 3,69 & 21,40 & 4,25 \\
Total & 23,56 & 4,42 & 22,71 & 4,35 & 23,14 & 4,38 \\
\hline
\end{tabular}

Nota. Médias seguidas de letras maiúsculas distintas e médias seguidas de letras minúsculas distintas diferem significativamente através da Análise de Variância, utilizando delineamento em medidas repetidas, ao nível de significância de 5\%.

A Tabela 4 mostra que, no tempo inicial, o grupo EM apresentou média significativamente maior do que no grupo $(F=8,89, p=0,005)$; no tempo final, essa diferença não foi significativa, havendo interação significativa entre os fatores tempo e grupo $(F=4,19, p=0,047)$. Em ambos os grupos não houve diferença significativa nas médias em relação aos tempos $(F=1,37, p=0,248)$.

Quanto aos resultados referentes ao estágio da Ação, o Grupo da EM obteve média inicial de 24, 44 (DP 3,82), final $24,41(D P 3,96)$ e total $24,43(D P 3,85)$, enquanto o grupo da Psicoeducação obteve média inicial de 22,24 (DP 4,46), final 24,19 (DP 4,43) e total 23,21 (DP 4,77). A amostra total obteve média inicial de 23,48 (DP 4,46), final 24,31 $(D P 4,13)$ e total 23,90 (DP 4,30). Em relação ao fator tempo, não houve diferença significativa entre a média total inicial e a média total final $(F=3,04, p=0,088)$. Quanto ao fator grupo, não houve diferença significativa entre as médias totais dos grupos $(F=1,18, p=0,283)$. Não foi constatada interação entre os fatores tempo e grupo ( $F=3,28, p=0,077)$.

Referente ao estágio da Manutenção, o grupo da EM obteve médias inicial de 19,15 (DP 6,35), final (19,07 (DP 4,98) e total 19,11 (DP 5,65), o grupo da Psicoeducação obteve médias inicial de 17,29 (DP 5,73), final 17,95 (DP 4,09) e total 18,46 (DP 5,37). A amostra total obteve médias inicial de 18,33 (DP 6,09), final 18,58 $(D P 4,60)$ e total $18,46(D P 5,37)$. Em relação ao fator tempo, não houve diferença significativa entre a média total inicial e a média total final $(F=0,17, p=0,682)$. Quanto ao fator grupo, não houve diferença significativa entre as médias totais dos grupos $(F=1,14, p=0,291)$. Não foi constatada interação entre os fatores tempo e grupo $(F=0,27, p=0,608)$. Resultados referentes à Prontidão para Mudança serão apresentados na Tabela 5.

Tabela 5

Média da Pontuação de Prontidão para Mudança com Análise de Variância em Medidas Repetidas

\begin{tabular}{lcccccc}
\hline \multirow{2}{*}{ Grupo } & \multicolumn{2}{c}{ Inicial } & \multicolumn{2}{c}{ Tempo } & \multicolumn{2}{c}{ Total } \\
\cline { 2 - 7 } & Média & Desvio-padrão & Média & Desvio-padrão & Média & Desvio-padrão \\
\hline Entrevista Motivacional & 9,21 & 2,29 & 9,25 & 2,07 & $9,23^{\mathrm{A}}$ & 2,16 \\
Psicoeducação & 7,25 & 2,50 & 7,95 & 1,98 & $7,60^{\mathrm{B}}$ & 2,26 \\
Total & 8,35 & 2,56 & 8,68 & 2,11 & 8,52 & 2,34
\end{tabular}

Nota. Médias seguidas de letras maiúsculas distintas e médias seguidas de letras minúsculas distintas diferem significativamente através da Análise de Variância, utilizando delineamento em medidas repetidas, ao nível de significância de 5\%.

Em relação ao fator tempo, não houve diferença significativa entre a média total inicial e a média total final $(F=1,6, p=0,212)$. Quanto ao fator grupo, independente do tempo, o grupo da EM teve média de prontidão para mudança significativamente maior $(F=8,7, p=0,007)$ que o grupo da Psicoeducação. Não foi constatada interação entre os fatores tempo e grupo $(F=1,3, p=0,260)$. 


\section{Discussão}

O objetivo principal das intervenções breves é atingir suas metas em um curto período de tempo. Neste estudo, inicialmente, o objetivo foi verificar se a Entrevista Motivacional, realizada em cinco sessões, poderia diminuir o consumo de drogas em adolescentes que cometeram ato infracional e que usavam drogas, bem como aumentar a sua motivação para mudança, diminuindo a ambivalência entre mudar ou não o comportamento de uso de drogas; e, para isso, a Psicoeducação seria uma técnica controle. No entanto, os resultados mostraram que tanto o grupo que recebeu a EM quanto o grupo que recebeu Psicoeducação, diminuíram o consumo de drogas demonstrando que as duas técnicas podem ser aplicadas à esta população.

O grupo da EM diminuiu o consumo de maconha e tabaco, e não houve redução significativa do uso de álcool. Gray, McCambridge e Strang (2005) realizaram um estudo com adolescentes com média de idade de 17 anos que receberam sessão única de $\mathrm{EM}$, e com outro grupo que não recebeu tratamento. $\mathrm{O}$ grupo que recebeu EM foi avaliado três meses depois e havia reduzido o uso de tabaco, mas não o uso de álcool e maconha. Outros estudos (Colby et al., 1998; Colby et al., 2005) relatam a redução do uso de tabaco e maconha, em sujeitos submetidos a EM (Andretta \& Oliveira, 2008; D'Amico et al., 2008; Martin, Copeland, \& Swift, 2005; White et al., 2006). A redução de álcool é relatada por diversos estudos em adolescentes tratados com EM (Borsari \& Carey, 2000; Monti et al., 1999; Tevyaw \& Monti, 2004), entretanto, neste estudo, esse efeito não foi constatado.

Resultados semelhantes foram encontrados em um estudo realizado por Marsden et al. (2006), com adolescentes entre 16 e 22 anos, usuários de múltiplas drogas, tratados com EM em dois grupos, com o mesmo número de sessões de tratamento. A diferença entre os grupos é que um deles não recebeu feedback da avaliação, princípio típico da EM, recebendo apenas informações sobre drogas. Não houve diferença nos resultados comparando as duas técnicas.

O grupo da Psicoeducação diminuiu o consumo de maconha e álcool. De acordo com um estudo de revisão sistemática realizada por Tait e Hulse (2003), intervenções breves em adolescentes alcançam índices de redução altas em álcool e pequenas reduções em tabaco e múltiplas drogas. Kaminer et al. (2002), compararam Terapia Cognitivo-Comportamental e Psicoeducação como controle, para reduzir o consumo de drogas em adolescentes, e na reavaliação, realizada após três meses, os índices de recaída foram menores no grupo de terapia, mas, na reavaliação realizada nove meses depois, os índices de recaídas, foram iguais nos grupos que receberam as duas técnicas.

No grupo da EM, a presença de comorbidades ao uso de drogas foi maior de TDAH que no grupo da Psicoeducação. O TOD também foi mais prevalente no grupo da EM, para fins diagnósticos, ele é suplantado pelo TC, e a prevalência deste foi semelhante nos dois grupos. Em um estudo realizado por Armstrong e Costello (2002), há o relato de que $60 \%$ dos adolescentes com diagnóstico de abuso ou dependência de substâncias têm algum diagnóstico de comorbidades, e a associação mais comum é com TDAH e com TOD, seguida por depressão. Neste mesmo estudo, está descrita a relação de uso de álcool com depressão e o uso de tabaco com transtornos de ansiedade, e de maconha com TDAH, resultado corroborado por outros estudos (French et al., 2008). Souza e Oliveira (2005), encontraram uma prevalência de TDAH $36,7 \%$ no grupo de usuários de drogas e $10 \%$ no grupo de adolescentes que não usavam drogas.

Outro estudo, realizado por Jané-Llopis e Matytsina (2006), descreve, além das associações já acima citadas, que existe associação entre o uso de drogas ilícitas com TC, além de sugerirem o tratamento de transtornos mentais como uma forma de prevenção ao uso de drogas.

Os resultados deste estudo, no grupo da EM, podem ter ficado prejudicados devido ao número maior de comorbidades. Shane, Jasiukaitis e Green (2003) realizaram um estudo para avaliar resultados de tratamento em adolescentes usuários de drogas com comorbidades, e verificaram que, em comparação com adolescentes sem comorbidades, apresentam resultados menores em índices de abstinência e redução de consumo de drogas. De acordo com Moreira e Soares (2004), a prevalência de comorbidades em dependentes químicos pode ser de $20-50 \%$, e o tratamento para dependência química pode não ser eficaz pela presença destas.

Alguns autores (Andretta \& Oliveira, 2008; Blanchard, Morgenstern, Morgan, Labouvie, \& Bux, 2003) descrevem a motivação para mudança de comportamentos relacionados ao consumo de drogas, em estágios que oscilam durante o processo. O grupo da EM estava menos pré-contemplativo comparado ao grupo da Psicoeducação, durante todo o processo. Houve redução na média de précontemplação entre a avaliação e a reavaliação para os dois grupos, no entanto, não houve diferença significativa entre os grupos. Desta forma, as duas técnicas foram efetivas para a entrada no processo de mudança. Quando o adolescente está pré-contemplativo, acredita que não tenha nenhum problema com o uso de drogas, e quando a pré-contemplação diminui, ele está menos resistente para o processo de mudança.

Na contemplação, o grupo da EM estava mais contemplativo que o grupo da Psicoeducação no início do processo, mas, ao término do processo, esse resultado não se manteve, pois o grupo da Psicoeducação teve médias semelhantes aos da EM. Significa dizer que o grupo da EM diminuiu a contemplação, ou seja, teoricamente os sujeitos deste grupo deram-se conta dos prejuízos causados pelo uso de drogas em sua vida, e estariam aptos a começar a fazer algo em relação à mudança, mas isso não ocorreu porque nos estágios de ação e manutenção, não houve mudança significativa. Erol e Erdogan (2008) ava- 
liaram os estágios motivacionais em uma população de adolescentes tabagistas na Turquia e encontraram redução na contemplação, aumento da ação e da manutenção nesta população após intervenção motivacional.

Não houve interação entre os fatores tempo e grupo nos estágios de ação e manutenção, e estes resultados, evidenciaram que, nesta população, estes formatos de intervenções não foram suficientes para que os adolescentes fizessem algo sobre mudar seu comportamento de uso de drogas. Apenas se deram conta dos prejuízos causados pelas substâncias, porém os resultados obtidos nos estágios ação e manutenção não se confirmaram pelos resultados na avaliação do consumo de drogas, pois houve redução nas duas intervenções.

Outros autores (Callaghan et al., 2008), descrevem a motivação para mudança de comportamentos, relacionado ao consumo de drogas, como uma medida única chamada de algoritmo de prontidão para mudança. $\mathrm{O}$ algoritmo é descrito como as médias da contemplação, da ação e da manutenção menos a média da pré-contemplação. Esse valor único seria o índice de prontidão para mudança, ou seja, esta pontuação definiria o estágio motivacional que o sujeito se encontra neste no momento. Pontuações até 8 indicam pré-contemplação, de 8 a 11, contemplação, e acima de 12, ação. Neste estudo, usando-se a medida do algoritmo, os participantes do grupo da Entrevista Motivacional entraram e saíram do processo contemplativos e os da Psicoeducação entraram e saíram pré-contemplativos, não apresentando modificação na prontidão para mudança entre a avaliação e a reavaliação.

Alguns autores relatam uma inadequação da escala URICA para adolescentes usuários de drogas, colocando em dúvida a confiabilidade e sensibilidade para esta população, sugerindo novos estudos (Callaghan et al., 2008; DiClemente, Schlund, \& Gemmel, 2004). Outros autores (Greenstein, Franklin, \& McGuffin, 1999) afirmam que a URICA apresenta boa consistência interna e que pode ser utilizada para o entendimento de pesquisas e tratamento para mudanças na adolescência.

Neste estudo, houve algumas limitações e a principal foi o baixo número de adolescentes encaminhados para tratamento. Em 4 anos de coleta de dados, chegaram ao serviço, especializado para o tratamento desta população, apenas 135 sujeitos, o que significa que apenas 1 adolescente a cada 10 dias foi encaminhado pela rede pública para o serviço ou, 3 a cada mês. Apesar dos critérios de inclusão, neste estudo, serem de conhecimento dos órgãos encaminhadores, o número de sujeitos encaminhados para avaliação foi pequeno, e, provavelmente, com uma amostra maior os resultados poderiam ser mais robustos, garantindo maior contribuição à ciência.

Ficam como sugestão para futuras pesquisas a filmagem das entrevistas para garantir maior fidedignidade da técnica e outras medidas, que não o auto-relato dos sujeitos, como a realização de exame toxicológico para verificação do uso de droga e controle das medicações utilizadas pelos sujeitos. Entretanto, o auto-relato do consumo de drogas ainda é muito utilizado em pesquisas com drogas, pois ainda é o método com menor custo para aferição e pode ser considerado uma medida confiável se comparado a exames químicos (McCambridge \& Strang, 2004).

\section{Referências}

American Psychiatric Association. (2002). DSM IV-TR: Manual diagnóstico e estatístico dos transtornos mentais (4. ed.). Porto Alegre, RS: Artes Médicas.

Andretta, I., \& Oliveira, M. (2005). A entrevista motivacional na adolescência. Revista de Psicologia Clínica, 17(2), 127139.

Andretta, I., \& Oliveira, M. (2008). Um estudo sobre os efeitos da entrevista motivacional em adolescentes infratores. Estudos de Psicologia (Campinas), 25(1), 45-53.

Armstrong, T., \& Costello, J. (2002). Community studies on adolescent substance use, abuse, or dependence and psychiatric comorbity. Journal of Consulting and Clinical Psychology, 70(6), 1224-1239.

Battjes, R. J., Gordon, M. S., O'Grady, K. E., Kinlock, T. W., Katz, E. C., \& Sears, E. A. (2004). Evaluation of a groupbased substance abuse treatment program for adolescents. Journal of Substance Abuse Treatment, 27(2), 123-134.

Becker, S., \& Curry, J. (2008). Outpatient interventions for adolescent substance abuse: A quality of evidence review. Journal of Consulting and Clinical Psychology, 76(4), 531-543.

Blanchard, K., Morgenstern, J., Morgan, T., Labouvie, E., \& Bux, D. (2003). Motivational subtipes and continuous measures of readiness for change: Concurrent and predictive validy. Psychology of Addictive Behaviors, 17(1), 56-65.

Borsari, B., \& Carey, K. (2000). Effects of brief motivational intervention with college student drinkers. Journal of Consulting and Clinical Psychology, 68(4), 217-228.

Brown, R., Ramsey, S., Strong, D., Myers, M., Kahler, C., Lejuez, C., et al. (2003). Effects of motivational interviewing on smoking cessation in adolescents with psychiatric disorders. Tobacco Control, 12(Suppl. 4), 3-10.

Callaghan, R., Taylor, L., Moore, B., Jungerman, F., Vilela, F., \& Budney, A. (2008). Recovery and Urica stange-of-changes scores in three marijuana treatments studies. Journal of Substance Abuse Treatment, 35(4), 419-426.

Carlini, E., Galduroz, J., Notto, R., \& Napo, S. (2002). I Levantamento Domiciliar Sobre o Uso de Drogas Psicotrópicas no Brasil. São Paulo, SP: Editora da Universidade Federal de São Paulo.

Colby, S. M., Monti, P., Barnett, N., Rohsenow, D., Weissman, K., Spirito, A., et al. (1998). Brief motivational interviewing in a hospital setting for adolescent smoking: A preliminary study. Journal of Consulting and Clinical Psychology, 66(3), 574-578

Colby, S. M., Monti, P., Tevyaw, T., Barnett, N., Spirito, A., Rohsenow, D., et al. (2005). Brief intervention for adolescent smokers in medical setting. Addictive Behaviors, 30(5), 865874.

Cunha, J. A. (2001). Manual da versão em português das Escalas Beck. São Paulo, SP: Casa do Psicólogo.

D’Amico, E., Miles, J., Stern, S., \& Meredith, L. (2008). Brief motivational intereview for teens at risk of substance use consequences: A randomized pilot study in a primary care clinic. Journal of Substance Abuse Treatment, 35(1), 53-61. 
Dennis, M. L., Titus, J. C., Diamond, G., Donaldson, J., Godley, S. H., Tims, F., et al. (2002). Steering Committee, The Cannabis Youth Treatment (CYT) Experiment: Rationale, study design, and analysis plans. Addiction, 97(Suppl. 1), S16-S34.

Diamond, G., Goodley, S., Liddle, H., Sampl, S., Webb, C., Tims, F., et al. (2002). Five outpatient treatment models for adolescent marijuana use: A description of the Cannabis Youth Treatment Interventions. Addiction, 97(Suppl. 1), S70-S83.

DiClemente, C., Schlundt, D., \& Gemmell, B. S. (2004). Readiness and stages of change in addiction treatment. American Journal of Addiction, 13(2), 103-119.

Dunn, C. W., Droesch, R. M., Johnston, B. D., \& Rivara, F. P. (2004). Motivational interviewing with injured adolescents in the emergency department: In-session predictors of change. Behavioural and Cognitive Psychotherapy, 32(1), 113-116.

Erol, S., \& Erdogan, S. (2008). Application of a stage based motivational interviewing approach to adolescent smoking cessation: The transtheoretical model-based study. Patient Education Counseling, 72(1), 42-48.

French, M., Zavala, S., McCollister, K., Waldron, H., Turner, C., \& Ozechowski, T. (2008). Cost-effectiveness analysis of four interventions for adolescents with a substance use disorder. Journal of Substance Abuse Treatment, 34(3), 272-281.

Gray, E., McCambridge, J., \& Strang, J. (2005). The effectiveness of motivational interviewing delivered by youth workers in reducing drinking, cigarette and cannabis smoking among young people: Quasi-experimental pilot study. Alcohol and Alcoholism, 40(6), 535-539.

Greenstein, D., Franklin, M., \& McGuffin, P. (1999). Measuring motivation to change: An examination of the Univesity of Rhode Island Change Assessment Questionaire (URICA) in adolescent sample. Psychotherapy, 36(1), 47-55.

Jané-Llopis, E., \& Matytsina, I. (2006). Mental health and alcohol, drugs and tobacco: A review of the comorbity between mental disorders and the use of alcohol, tobacco e illicit drugs. Drug and Alcohol Review, 25(6), 515-536.

Kaminer, Y., Burleson, J., \& Goldberger, R. (2002). Cognitivebehavioral coping skills and psychoeducation therapies for adolescent substance abuse. The Journal of Nervous and Mental Disease, 190(11), 737-745.

Knapp, P., Luz, E., \& Baldisserotto, G. (2001). Terapia cognitiva no tratamento da dependência química. In B. Rangè (Ed.), Psicoterapias cognitivo-comportamentais: Um diálogo com a Psiquiatria. Porto Alegre, RS: Artes Médicas, pp. 332350)

Lawendowski, L. (1998). A motivational intervention for adolescent smokers. Preventive Medicine, 27(5), A29-A46.

Lincourt, P., Kuettel, T., \& Bombardier, C. (2002). Motivational interviewing in a roup setting with mandated clients. A pilot study. Addicitve Behaviors, 27(3), 381-391.

Marsden, J., Stillwell, G., Barlow, H., Boys, A., Taylor, C., Hunt, N., et al. (2006). An evaluation of a brief intervention among young ecstasy and cocaine users: No effect on substance and alcohol outcomes. Addiction, 101(7), 1014-1026.

Martin, G., \& Copeland, J. (2008). The adolescent cannabis check-up: Randomized of a brief intervention for young cannabis users. Journal of Substance Abuse Treatment, 34(4), 404-414.

Martin, G., Copeland, J., \& Swift, W. (2005). The adolescent cannabis check-up: Feasibility of a brief intervention for young cannabis users. Journal of Substance Abuse Treatment, 29(3), 207-213.
McCambridge, J., \& Strang, J. (2004). The efficacy of singlesession motivational interviewing in reducing drug consumption and perceptions of drug-related risk and harm among young people: Results from a multi-site cluster randomized trial. Addiction, 99(1), 39-52.

Miller, W. R., \& Rollnick, S. (2001). Entrevista motivacional: Preparando pessoas para a mudança. Porto Alegre, RS: Artes Médicas.

Monti, P., Colby, S. M., Barnett, N., Spirito, A., Rohsenow, D., Myers, M., et al. (1999). Brief intervention for harm reduction with alcohol positive older adolecents in a hospital emergency department. Journal of Consulting and Clinical Psychology, 67(6), 989-994.

Moreira, E. C., \& Soares, G. H. G. (2004). Comorbidade e dependência química: Repercussões na adesão ao tratamento e evolução clínica. In L. A. Tavares, A. Riva, A. N. Fo, E. MacRae, L. A. Tavares, \& O. S. Ferreira (Eds.), Drogas: Tempos, lugares e olhares sobre seu consumo. Salvador, BA: Editora da Universidade Federal da Bahia.

Muck, R., Zempolich, K. A., Titus, J. C., Fishman, M., Godley, M. D., \& Schwebel, R. (2001). An overview of the effectiveness of adolescent substance abuse treatment models. Youth Society, 33(2), 143-168.

Sampl, S., \& Kadden, R. (2001). CYT: Canabis Youth Treatment. Madison, CT: Daniel Hand High School.

Secretaria Nacional Antidrogas. (2004). Maconha: Informações para os adolescentes (5. ed.). Brasília, DF: Autor.

Shane, P., Jasiukaitis, P., \& Green, R. (2003). Treatment outcomes among adolescents with substance abuse problems: The relationship between comorbities and pos-treament substance involvement. Evaluation and Program Planning, 26, 393-402.

Smith, T., Sell, S., Rodman, J., \& Reynolds, L. (2006). Reducing adolescent substance abuse and delinquency pilot research of a family-oriented psychoeducation curriculum. Journal of Child and Adolescent Substance Abuse, 15(4), 105-115.

Souza, C. C., \& Oliveira, M. O. (2005). Transtorno de déficit de atenção e hiperatividade. Arquivos Brasileiros de Psiquiatria, Neurologia e Medicina Legal, 99(3), 45-56.

Stein, L., Monti, P., Lebeau-Craven, R., Miranda, R., Colby, S., Barnett, N., et al. (2006). Enhancing substance abuse treatment engagement in incarcerated adolescents. Psychologic Services, 3(1), 25-34.

Szupszynski, K., \& Oliveira, M. O. (2008). Adaptação brasileira da University of Rhode Island Change Assessment (URICA) para usuários de substâncias ilícitas. Psico-USF, 13(1), 31-39.

Tait, R., \& Hulse, G. (2003). A systematic review of the effectiveness of brief interventions with substance using adolescents by type of drug. Drug and Alcohol Review, 22(3), 337-346.

Tevyaw, T., \& Monti, P. (2004). Motivacional enhancement and other brief interventions for adolescent substance abuse: Foundations, applications and evaluations. Addiction, 99(2), 63-75.

White, H., Morgan, T., Pugh, L., Celinska, K., Labouvie, E., \& Pandina, R. (2006). Evaluation two brief substance use interventions for mandated college students. Journal of Studies on Alcohol, 67(2), 309-317. 